\title{
Optimization of geometry for the lateral buckling process of a cantilever beam
}

\author{
R. Drazumeric, F. Kosel \& T. Kosel \\ Faculty of Mechanical Engineering, University of Ljubljana, Slovenia
}

\begin{abstract}
Using the large displacement theory (theory of the third order according to Chwalla), this paper deals with the lateral buckling process of a slender, elastic cantilever beam with a changeable height of a rectangular cross section and represents it with a system of nonlinear differential equations. Based on a mathematical model of the lateral buckling process, which considers the geometric and boundary conditions, an optimal geometry of a cantilever beam is obtained using the calculus of variation. A comparison between the properties of the beam with optimized geometry and those of a referential beam with a constant cross section is shown. The result of the optimization process is, besides a higher critical load, a higher carrying capacity of the optimal geometry beam in the postbuckling region. For a verification of the theoretical results an experiment of the lateral buckling process had been done.

Keywords: elastic stability, lateral buckling, geometry optimization, calculus of variation, large displacement theory.
\end{abstract}

\section{Introduction}

Lateral buckling of a bent cantilever beam is a stability problem, where a small lateral disturbance in an unstable equilibrium state produces a spatial deflection of the beam, and as a result a combination of bending and torsional load appears. This transition causes an additional load on the beam, so in the design process it should be ensured that the load does not exceed its critical value. That is the reason why, in cases of slender elements where the stability limit is the main criterion, the load carrying capacity of the material is poorly exploited. One possible way of increasing the stability limit and better exploit the load carrying capacity of the element is to optimize its geometry. 
The purpose of this paper is to represent an analytical method of geometry optimization of a bent cantilever beam and its numerical and experimental verification. Detailed theoretical studies of the geometry optimization method were previously presented in the paper [1]. The method of geometry optimization can be further applied to problems of buckling with one-dimensional elastic elements for various conditions and loads, as shown in dissertation [2].

\section{Mathematical model of the lateral buckling process}

The model of the lateral buckling process on a slender cantilever beam with a rectangular cross section is obtained using the large displacement theory, and is based on the assumption that stress in the loaded beam lies in the elastic region of the material.

Figure 1 shows a buckled cantilever beam. The spatial deformation state of the beam is determined with three functions:

- the lateral deflection of the neutral axis $v$,

- the vertical deflection of the neutral axis $w$,

- the rotation of the cross section $\vartheta$.

Quantities $(\bar{x}, \bar{v}, \bar{w})$, which are used to describe the position of the elastic curve in space, are given per unit of the length of the elastic curve $l$, so they are non-dimensional. The position of the cross section is given with the unit vectors, which represent the orthonormal basis of the subsidiary coordinate system:

- the tangent vector of the elastic curve $\vec{e}_{\xi}=\left(e_{\xi_{1}}, e_{\xi_{2}}, e_{\xi 3}\right)$,

- the vector of the symmetry axis of the cross section in the thickness direction $\vec{e}_{\eta}=\left(e_{\eta 1}, e_{\eta 2}, e_{\eta 3}\right)$,

- the vector of the symmetry axis of the cross section in the height direction $\vec{e}_{\zeta}=\left(e_{\zeta 1}, e_{\zeta 2}, e_{\zeta 3}\right)$.

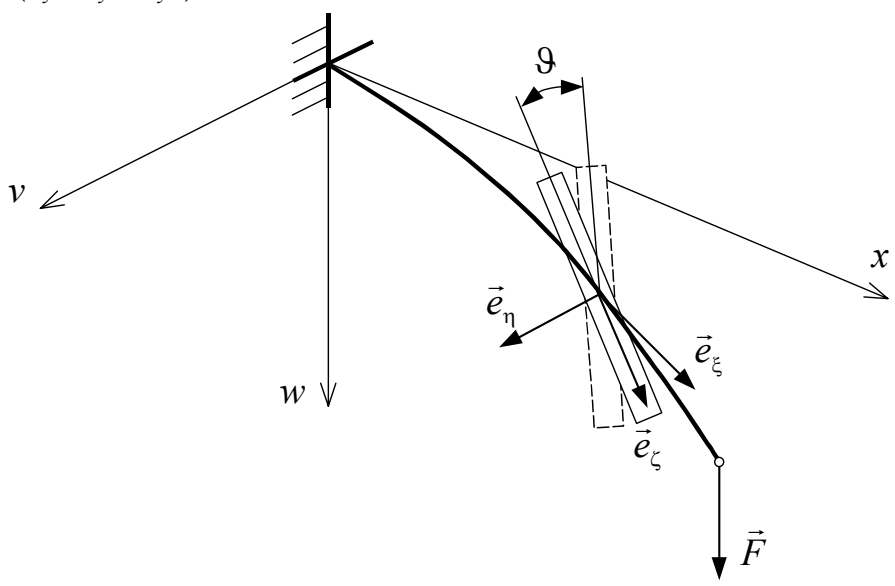

Figure 1: An elastic curve of a cantilever beam in the deflected form. 
The results of the lateral buckling of the beam with optimized geometry will be compared with the results of lateral buckling of the referential beam with a constant cross section. For this purpose we define appropriate non-dimensional parameters that will be used for the analysis of the lateral buckling process with respect to relative properties of the referential beam.

- Relative height of the cross section

$$
\bar{h}(\bar{x})=h(\bar{x}) / h_{0},
$$

where $h(\bar{x})$ is height of the cross section in point $\bar{x}$, and $h_{0}$ is height of the cross section of the referential beam.

- Relative thickness of the cross section

$$
\bar{t}(\bar{x})=t(\bar{x}) / t_{0}=1
$$

where $t(\bar{x})$ is thickness of the cross section, which is constant along the beam in considered case and is equal to thickness of the cross section of the referential beam $t_{0}$.

- Relative cross-sectional area

$$
\bar{A}(\bar{x})=A(\bar{x}) / A_{0}=\bar{h}(\bar{x}),
$$

where $A(\bar{x})$ is cross-sectional area in point $\bar{x}$, and $A_{0}$ is cross-sectional area of the referential beam.

- Relative moment of inertia of the rectangular cross section

$$
\bar{I}(\bar{x})=I_{t}(\bar{x}) / I_{t 0}=I_{\zeta}(\bar{x}) / I_{\zeta 0}=\bar{h}(\bar{x}),
$$

where the following relations are valid for a slender rectangular cross section: $I_{t}(\bar{x})=h(\bar{x}) t_{0}^{3} / 3, I_{t 0}=h_{0} t_{0}^{3} / 3$ and $I_{\zeta}(\bar{x})=h(\bar{x}) t_{0}^{3} / 12, I_{\zeta 0}=h_{0} t_{0}^{3} / 12$.

- Relative load

$$
\bar{F}=F / F_{0},
$$

where $F$ is a load on the free end of the beam, and $F_{0}$ is the critical buckling load of the referential beam. Critical buckling load $F_{0}$ is according to Timoshenko and Gere [3] given with the expression:

$$
F_{0}=\frac{4.013}{l^{2}} \sqrt{E G I_{\zeta 0} I_{t 0}} .
$$


The mathematical model is based on the relations between loads and deformations, which appear in the lateral buckling process. We introduce the expressions for the inner moments, corresponding deformational quantities, properties of the material and the defined non-dimensional parameters into these relations. After appropriate transformation we can write a system of nonlinear differential equations in the final non-dimensional form:

$$
\begin{gathered}
\vartheta^{\prime}=\frac{\bar{F} \cdot 4.013}{\bar{h}} \sqrt{\frac{1+v}{2}}\left[\left(\bar{v}_{1}-\bar{v}\right) e_{\xi 1}-\left(\bar{x}_{1}-\bar{x}\right) e_{\xi 2}\right] \sqrt{1+\bar{v}^{\prime 2}+\bar{w}^{\prime 2}}, \\
\bar{v}^{\prime \prime}=\frac{\bar{F} \cdot 4.013}{\bar{h}} \sqrt{\frac{2}{1+v}}\left[\left(\bar{v}_{1}-\bar{v}\right) e_{\zeta 1}-\left(\bar{x}_{1}-\bar{x}\right) e_{\zeta 2}\right]{\sqrt{1+\bar{v}^{\prime 2}+\bar{w}^{\prime 2}}}^{3} e_{\zeta 3}, \\
\bar{w}^{\prime \prime}=-\frac{\bar{F} \cdot 4.013}{\bar{h}} \sqrt{\frac{2}{1+v}}\left[\left(\bar{v}_{1}-\bar{v}\right) e_{\zeta 1}-\left(\bar{x}_{1}-\bar{x}\right) e_{\zeta 2}\right]{\sqrt{1+\bar{v}^{\prime 2}+\bar{w}^{\prime 2}}}^{3} e_{\zeta 2},
\end{gathered}
$$

where $v$ is Poisson's coefficient of the material, $\bar{x}_{1}$ is value of the variable $\bar{x}$ on the free end and $\bar{v}_{1}$ is value of the lateral displacement on the free end. The value $\bar{x}_{1}$ is determined with the expression for the elastic curve length in the normal form:

$$
\int_{0}^{\bar{x}_{1}} \sqrt{1+\bar{v}^{\prime 2}+\bar{w}^{\prime 2}} d \bar{x}=1 .
$$

Boundary conditions for the system of differential equations, eqns (7), (8), and (9), are: $\vartheta(0)=0, \bar{v}(0)=0, \bar{w}(0)=0, \bar{v}^{\prime}(0)=0$ and $\bar{w}^{\prime}(0)=0$.

\section{Geometry optimization}

Definition of the geometry optimization problem is: for a slender cantilever beam with a rectangular cross section and fixed length we are trying to find an appropriate longitudinal shape of the beam that would give maximal critical buckling load under the condition that the volume of the optimized beam is equal to the volume of the referential beam with a constant cross section.

\subsection{Variational problem}

The unstable state, which appears when the load reaches its critical value $\bar{F}_{c}$, represents a limit state of the buckling process where a small disturbance causes transformation of an element from an unstable to a new stable form. So in this limit state we can substitute functions used in the model of the lateral buckling process with their differentials - we linearize the mathematical model. 
The linearized model is written using a new variable $\bar{u}=1-\bar{x}$ and it can be transformed into an ordinary differential equation of the second order for the function $\vartheta(\bar{u})$ :

$$
\bar{h}^{2} \vartheta^{\prime \prime}+\bar{h} \bar{h}^{\prime} \vartheta^{\prime}+\bar{F}_{c}^{2} \cdot 4.013^{2} \cdot \bar{u}^{2} \vartheta=0 .
$$

It turns out that in the case of optimal geometry the following boundary conditions are valid for eqn $(11): \bar{h}(0)=0 \quad\left(\vartheta^{\prime}(0) \neq 0\right), \vartheta(1)=0$.

The optimization is based on the geometric condition of unchanging volume of the cantilever beam, which is expressed with an equation:

$$
\int_{0}^{1} \bar{h}(\bar{u}) d \bar{u}=1 .
$$

Eqn (11) can be represented in two ways: as linear differential equation of the second order for the function $\vartheta(\bar{u})$ or as differential equation of the first order for the function $\bar{h}(\bar{u})$. We can solve the differential equation for the function $\bar{h}(\bar{u})$ and introduce this solution into eqn (12), so the relative critical load could be written in the following form:

$$
\bar{F}_{c}=\frac{1}{4.013 \cdot \sqrt{2} \cdot J(\vartheta)},
$$

where $J(\vartheta)$ represents a functional:

$$
J(\vartheta)=\int_{0}^{1} \sqrt{\frac{-\int_{0}^{\bar{u}} \bar{y}^{2} \vartheta \vartheta^{\prime} d \bar{y}}{\vartheta^{\prime 2}}} d \bar{u} .
$$

Using eqn (13) we can write the final form of solution of optimization problem:

$$
\bar{A}(\bar{u})=\frac{\sqrt{\frac{-\int_{0}^{\bar{u}} \bar{y}^{2} \vartheta \vartheta^{\prime} d \bar{y}}{\vartheta^{\prime 2}}}}{J(\vartheta)} .
$$

The relative critical load $\bar{F}_{c}$ will be maximal if the value of functional $J(\vartheta)$ is minimal. So we define the variational problem as follows: among all function $\vartheta(\bar{u})$ that are in the interval $[0,1]$ continuous and continuously differentiable, we are looking for the one that would give a minimum value to functional $J(\vartheta)$. 
The variational problem was solved using the expression for function $\vartheta(\bar{u})$ in the form of a series, Vidav [4], that fulfills the prescribed boundary conditions:

$$
\vartheta=1-\bar{u}+\sum_{k=1}^{k=n} \alpha_{k}\left(\bar{u}^{k}-\bar{u}^{k+1}\right) .
$$

Using this expression, we transform the functional, eqn (14), into a function of $n$ variables: $J(\vartheta)=g\left(\alpha_{1}, \alpha_{2}, \ldots, \alpha_{n}\right)$. The necessary condition for the minimum of function of $n$ real variables is represented with the system of $n$ nonlinear equations, Arora [5]:

$$
\frac{\partial g}{\partial \alpha_{k}}=0 ; k=1,2, \ldots, n
$$

The nonlinear system of equations was solved numerically, according to Hoffman [6].

\subsection{Results of geometry optimization}

The main result of the geometry optimization process is maximal relative critical load: $\bar{F}_{c \max }=1.235$. Corresponding solution of the variational problem is shown in Figure 2.
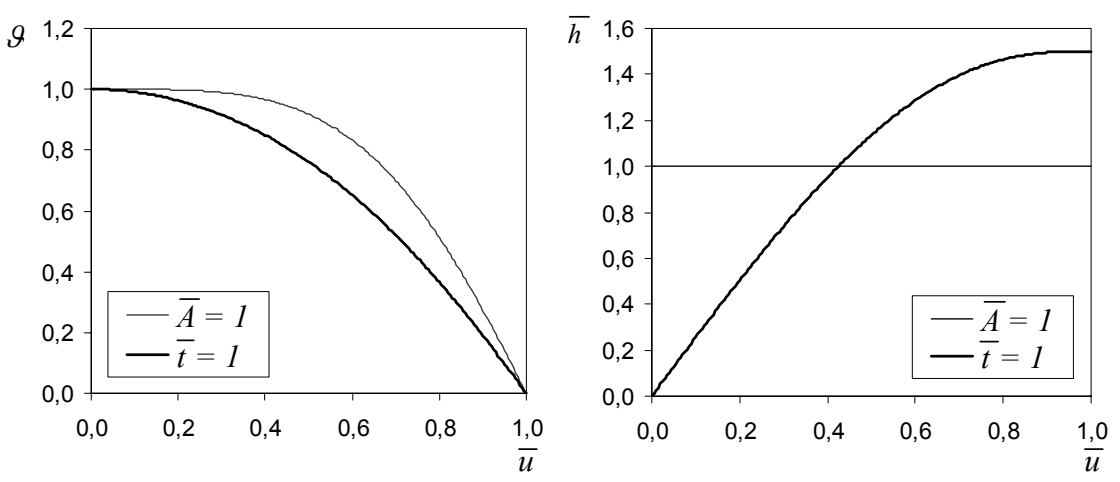

Figure 2: Solution of variational problem.

In the root of the solution of the variational problem $(\bar{u}=0)$ singularity appears, cross-sectional area is equal to zero. This means, that shear stress, which is present before the lateral buckling of a beam, is not limited in the point of singularity. Beside this, an assumption of a slender beam is violated around the singularity point. For experimental use of the results, a shear stress constraint was included in the optimization procedure and appropriate values of geometry 
and material parameters of the referential beam ensured that all assumptions that were made in the mathematical model of the lateral buckling process, were fulfilled.

\section{Results for postbuckling region}

In the geometry optimization procedure we discuss the behaviour of the beam in the unstable state of the lateral buckling process. To represent the behaviour of the cantilever beam in the postbuckling region, we should find a solution of the system of nonlinear differential equations, eqns $(7,8,9)$, which represents the mathematical model of the lateral buckling process by the large displacement theory. The system of equations is solved numerically with the Runge-Kutta method, Hoffman [6].

In the unstable state of the lateral buckling process, the lateral displacement of the free end rapidly increase, Figure3. With higher values of the relative load the lateral displacement is converging to the finite value. Figure 3 also shows the maximal nondimensional reference stress on the clamped end $\sigma_{\max }^{*}$, which is obtained by the deformation energy theory, Skerlj [7], and given with the following expression:

$$
\sigma_{\max }^{*}=\frac{\bar{F} \cdot 4.013 \cdot \bar{v}_{1}}{\bar{h}(0)} \sqrt{\frac{1+v}{2}}
$$

In case of optimal geometry, maximal reference stress in the postbuckling region increases more slowly, despite higher critical buckling load. Values of the actual reference stress are determined with the appropriate selection of the geometry and material parameters of the referential beam.
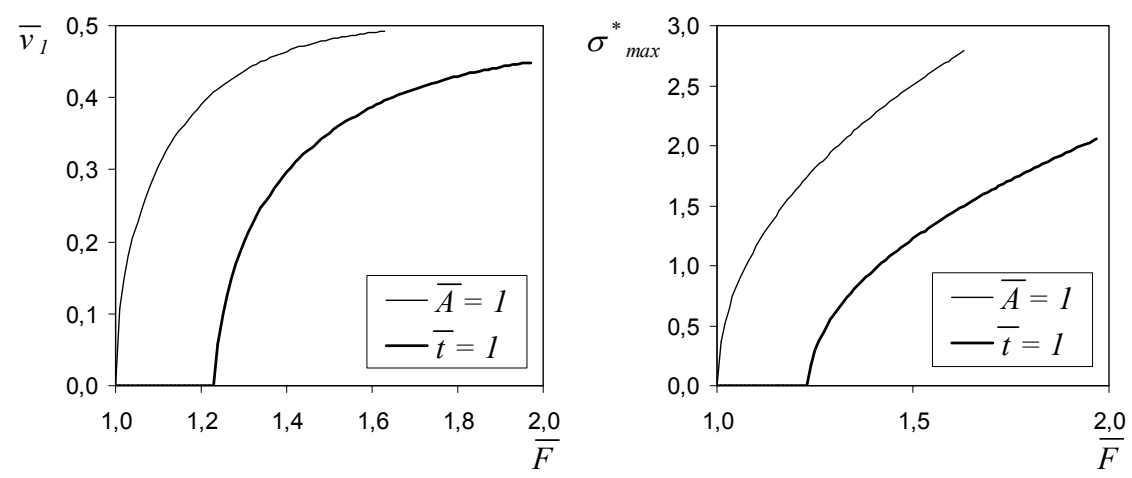

Figure 3: Results for postbuckling region. 


\section{Experimental verification of the theoretical results}

An experiment of the lateral buckling process was realized for the beam with optimized geometry and the referential beam with a constant cross section. Results of the experiment are measured lateral displacements of the free end for given loads. Appropriate selection of the material and geometry parameters ensures that stress in the loaded beam lies in the elastic region. In order to keep postbuckling stresses in the elastic region we have used steel with high carrying capacity for the experiment.

In theoretical calculations we need a value of Young's modulus of elasticity of the material $E$. Modulus of elasticity have been obtained from bending test, Figure 4, where surface characteristics have crucial influence on mechanical properties - similar to buckling process. Modulus is determined with relation between load and deformation at bending test:

$$
E=\frac{F}{\hat{x}_{f} I_{\zeta 0}} \frac{l^{3}}{48},
$$

where $\hat{x}_{f}$ is average value of measured displacements $f$, Figure 4 .

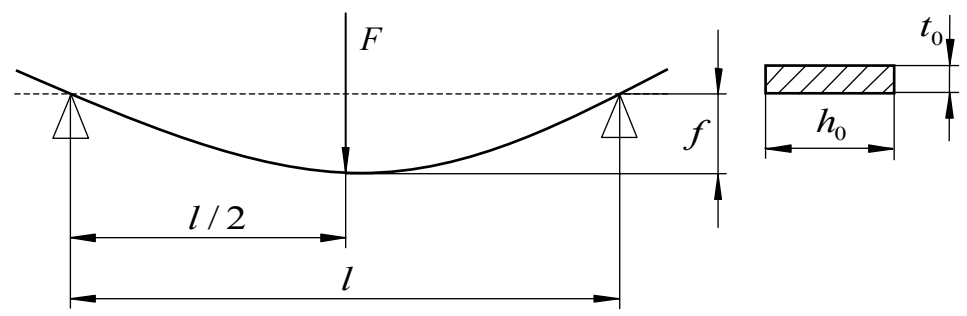

Figure 4: Bending test.

Experiment of the lateral buckling process showed an existence of initial curvature of the tested beams. This property causes different displacements depending on which side the beam buckles. Results that correspond to the buckling on the side of initial curvature are characterized as results A and those that correspond to the opposite side as results B. Comparison between the theoretical and the experimental results was made just for the case $\mathrm{B}$, because the unstable state is present only in that case.

Theoretical and experimental results of tested beams are shown in Figure 5 and Tables 1,2, where $\hat{x}_{F}$ is average value of corresponding load and $\delta F$ is relative load error. In the Figure 5 can be seen that at higher postbuckling load results A and results B converge to the same value. Analysis of the results shows that in the case of referential beam, theoretical and experimental results are in good agreement and in the case of optimized beam, experimental load is slightly 
higher than theoretical. The main reason for existing errors is that the optimized geometry exposes influence of hardened surface of the material on the beam.
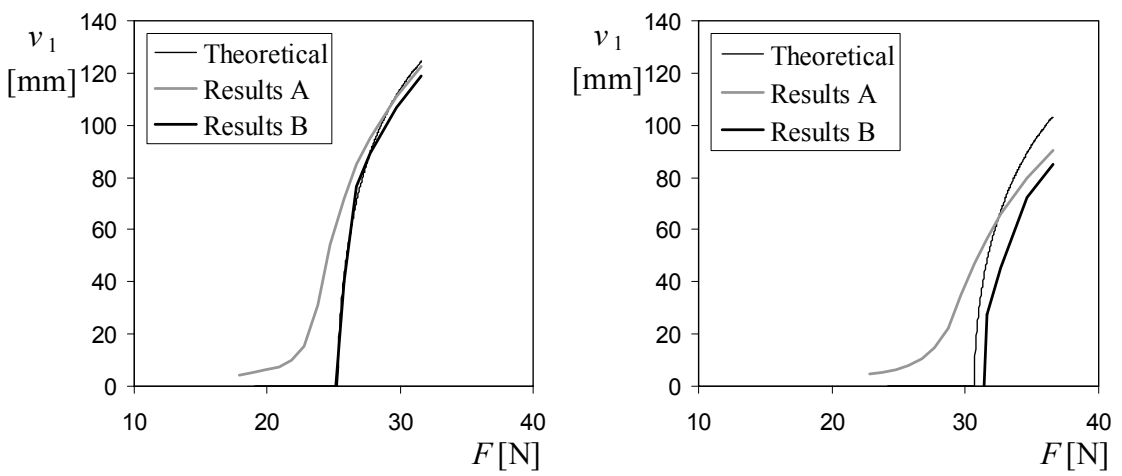

Figure 5: Theoretical and experimental results for the referential beam and the beam with optimized geometry.

Table 1: Comparison between theoretical and experimental results for the referential beam.

\begin{tabular}{|c|c|c|c|}
\hline$v_{1}[\mathrm{~mm}]$ & $F[\mathrm{~N}]$ & $\hat{x}_{F}[\mathrm{~N}]$ & $\delta F[\%]$ \\
\hline 0 & 25,33 & 25,20 & $-0,52$ \\
\hline 20 & 25,43 & 25,48 & 0,20 \\
\hline 40 & 25,74 & 25,75 & 0,04 \\
\hline 60 & 26,30 & 26,29 & $-0,04$ \\
\hline 80 & 27,18 & 27,01 & $-0,63$ \\
\hline
\end{tabular}

Table 2: Comparison between theoretical and experimental results for the beam with optimized geometry.

\begin{tabular}{|c|c|c|c|}
\hline$v_{1}[\mathrm{~mm}]$ & $F[\mathrm{~N}]$ & $\hat{x}_{F}[\mathrm{~N}]$ & $\delta F[\%]$ \\
\hline 0 & 30,68 & 31,46 & 2,48 \\
\hline 20 & 30,83 & 31,59 & 2,41 \\
\hline 40 & 31,32 & 32,32 & 3,09 \\
\hline 60 & 32,22 & 33,68 & 4,33 \\
\hline 80 & 33,67 & 35,75 & 5,82 \\
\hline
\end{tabular}

\section{Conclusions}

The contribution presents an analytical approach to geometry optimization of lateral buckling process of a slender, elastic cantilever beam. Critical buckling load is considerably higher in the case of the optimized beam. 
With the solution of the system of nonlinear differential equations, representing the mathematical model of the lateral buckling process by the large displacement theory, the stress - strain state of the beam has been quantified. With the appropriate selection of values of the referential beam parameters, the optimized beam shows certain carrying capacity also in the postbuckling region, despite a higher critical buckling load. The solution of the optimization problem and the solution of the system of nonlinear differential equations are, considering certain conditions, valid in general, regardless of properties of the referential beam with constant cross section.

Experiment verification of the results shows that in the case of referential beam theoretical and experimental results are in good agreement. In the case of optimized beam combined effect of hardened surface and optimized geometry causes slightly higher experimental loads.

\section{References}

[1] Drazumeric, R. \& Kosel, F., Optimization of geometry for lateral buckling process of a cantilever beam in the elastic region. Thin-Walled Structures, 43(3), pp. 515-529, 2005.

[2] Drazumeric, R., Optimization of geometry for lateral buckling problems of one-dimensional elements, PhD thesis, University of Ljubljana, Faculty of Mechanical Engineering, 2001.

[3] Timoshenko, S.P. \& Gere, J.M., Theory of elastic stability, McGraw-Hill: New York, 1961.

[4] Vidav, I., Variacijski racun, Drustvo matematikov, fizikov in astronomov Slovenije, 1991.

[5] Arora, J.S., Introduction to optimum design, McGraw-Hill: New York, 1989.

[6] Hoffman, J.D., Numerical methods for engineers and scientists, McGrawHill: New York, 1992.

[7] Skerlj, M., Mehanika-trdnost, University of Ljubljana, Faculty of Mechanical Engineering, 1988. 